\title{
Switching the Twist in X Rays with Magnets
}

\author{
Scientists create a pattern of nanomagnets-called an artificial spin \\ ice-that can control the orbital angular momentum of a scattered $\mathrm{x}$-ray \\ beam.
}

By Chen-Ting Liao, Carlos Hernández-García, and Margaret M. Murnane

$\Lambda$ beam of $x$ rays with a spiral wave front can be used to characterize spin and chiral textures, such as magnetic vortices, hedgehogs, and skyrmions, inside a material. However, generating these twisted $x$ rays isn't trivial. Over the past two years, scientists have achieved this goal by designing and fabricating extremely precise $x$-ray optics devices $[1,2]$. These devices are passive, meaning that their effect on light is

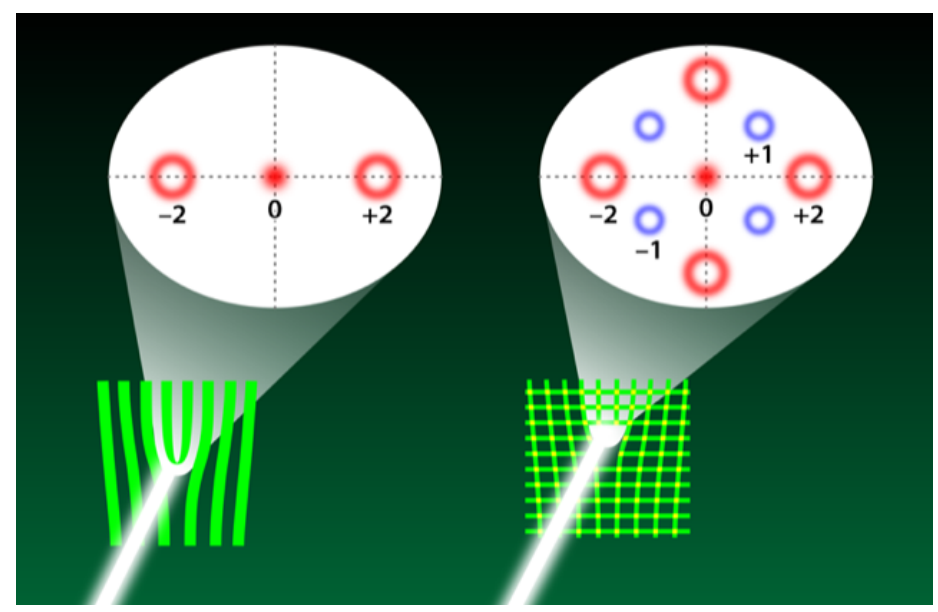

Figure 1: Two strategies for generating orbital angular momentum (OAM) in light. The left side shows a 1D-like microstructure, called a fork-dislocation hologram, which is commonly used to generate OAM beams in visible light. The right side shows a 2D nanomagnet array, called an artificial spin ice, which was used by Woods, Chen, and colleagues to generate $x$-ray OAM beams. Structural (charge) scattering generates even-order beams (red doughnuts), while magnetic (spin) scattering generates odd-order beams (blue doughnuts).

Credit: APS/Alan Stonebraker fixed, like that of a lens or a mirror. Now Justin Woods of the University of Kentucky, Xiaoqian Chen of Brookhaven National Laboratory, New York, and colleagues have realized an active device that can control the properties of an $\mathrm{x}$-ray beam on the fly [3]. The team used an engineered nanomagnet array-called an artificial spin ice-that twists $x$ rays by different amounts. By changing the temperature or by using an external magnetic field, the team showed that they could control the amount of twisting and the direction of the outgoing beams. This flexibility could be advantageous for probing or controlling electronic and magnetic systems.

Scientists' ability to discover and observe fundamental processes in nature has been historically connected to their capability to harness the properties of light. In the early 1960s, the development of the laser and nonlinear optics allowed scientists to exquisitely control the wavelength (energy), polarization (spin angular momentum), wave vector (linear momentum), amplitude, and phase of light. In the 1990s, scientists realized that light beams can also possess a property-the orbital angular momentum (OAM)-which involves the rotation of the beam's phase around its central axis (OAM) [4].

The OAM of light is associated with spiral wave fronts of an electromagnetic wave. Different modes exist, distinguished by the "light topological charge," which corresponds to the number of spirals in the spatial evolution of the phase. OAM is of fundamental interest to the manipulation of light, impacting current and future applications that include optical manipulation of particles, super resolution imaging, optical metrology, optical communications, and quantum computing 
$[5,6]$. Moreover, by extending the OAM of light to shorter wavelengths it should be possible to more sensitively probe chiral and topological structure in matter.

However, imprinting OAM onto ultraviolet and $\mathrm{x}$-ray beams is technically challenging. In the extreme ultraviolet (EUV) region of the electromagnetic spectrum, exciting advances in high harmonic generation have made it possible to generate beams with high spatial and temporal coherence and to tailor their properties by sculpting the driving laser field. These advances have enabled subwavelength imaging [7] as well as the generation of EUV OAM beams $[8,9]$. In the $x$-ray regime, any optical device requires ultraprecise fabrication-to within a fraction of the $x$-ray wavelength, corresponding to less than an angstrom $\left(10^{-10} \mathrm{~m}\right)$. Researchers have designed diffraction gratings that can produce x-ray OAM beams $[1,2]$. However, generating programmable OAM beams in the $\mathrm{x}$-ray region had not previously been done.

In their work, Woods, Chen, and colleagues have engineered and tested a new approach for generating $\mathrm{x}$-ray beams carrying OAM [3]. They fabricated a device based on an artificial spin ice, which consisted of nickel-iron nanomagnets arranged in a two-dimensional lattice. The team designed their spin-ice array so that it contained a specific topological defect consisting of a double dislocation, which looks like a hole in the strings of a tennis racket. The fundamental working principle of their artificial spin ice can be understood as a "fork-dislocation hologram," which is a slightly warped diffraction grating that has been used since the 1990s to generate visible OAM beams [10]. Similarly, when an x-ray beam struck the spin-ice dislocation, multiple $x$-ray beams (diffraction modes) scattered out in different directions, each with a different amount of OAM-or light topological charge (Fig. 1).

Interestingly, the defect in the artificial spin ice had two separate scattering effects on an incoming $x$-ray beam. The double dislocation in the arrangement of electric charges had a "structural topological charge" of 2, which meant it generated diffraction modes with even-order OAM (light topological charge of $\pm 2, \pm 4$, etc.). By contrast, the dislocation in the magnetic spins had a topological charge of 1 , which meant it generated odd-order OAM modes (light topological charge of $\pm 1, \pm 3$, etc.) in $x$ rays that were resonant with an absorption line of iron. However, this magnetic scattering was only possible when the artificial spin ice had an antiferromagnetic (antiparallel) ordering of its spins. Thus, the researchers could turn off this scattering by inducing an antiferromagnetic-to-paramagnetic phase transition. This transition was dependent on the temperature and on the external magnetic field. The team showed that they could switch the OAM of the outgoing $x$-ray beam from a mix of odd and even orders to only even orders-either by increasing the temperature from $270 \mathrm{~K}$ to $380 \mathrm{~K}$ or by applying a magnetic field.

The use of artificial spin ice with structural and magnetic topological defects offers new capabilities for $\mathrm{x}$-ray OAM beam generation. Adaptive optical components that can control the OAM properties of $x$-ray light can enhance our ability to probe and image chiral and topological nanostructures, chiral molecules used in medicines, and magnetic and other materials relevant to nanotechnologies. More opportunities to manufacture other types of active x-ray devices will come from emerging nanomaterials and nanodevices that are reconfigurable or controllable through phase transitions. In combination with devices that create structured EUV and soft $x$-ray beams through extreme nonlinear optics, these advances can greatly expand the capabilities of structured, short-wavelength light for capturing the time-resolved dynamics and functions of topological structures.

Correction (16 March 2021): The text was revised to include the contribution of Xiaoqian Chen, who was also a lead author of the study.

Chen-Ting Liao: JILA - Department of Physics, University of Colorado and NIST, Boulder, CO, USA

Carlos Hernández-García: Department of Applied Physics, University of Salamanca, Salamanca, Spain

Margaret M. Murnane: JILA - Department of Physics, University of Colorado and NIST, Boulder, CO, USA

\section{REFERENCES}

1. J. C. T. Lee et al., "Laguerre-Gauss and Hermite-Gauss soft $\mathrm{x}$-ray states generated using diffractive optics," Nat. Photon. 13, 205 (2019).

2. L. Loetgering et al., "Generation and characterization of focused helical x-ray beams," Sci. Adv. 6, eaax8836 (2020). 
3. J. S. Woods et al., "Switchable x-ray orbital angular momentum from an artificial spin ice," Phys. Rev. Lett. 126, 117201 (2021).

4. L. Allen et al., "Orbital angular momentum of light and the transformation of Laguerre-Gaussian laser modes," Phys. Rev. A 45, 8185 (1992).

5. Y. Shen et al., "Optical vortices 30 years on: OAM manipulation from topological charge to multiple singularities," Light Sci. Appl. 8, 90 (2019).

6. B. Wang et al., "Coherent Fourier scatterometry using orbital angular momentum beams for defect detection," Opt. Express 29, 3342 (2021).
7. D. F. Gardner et al., "Subwavelength coherent imaging of periodic samples using a $13.5 \mathrm{~nm}$ tabletop high-harmonic light source," Nat. Photon. 11, 259 (2017).

8. C. Hernández-García et al., "Attosecond extreme ultraviolet vortices from high-order harmonic generation.," Phys. Rev. Lett. 111, 083602 (2013).

9. L. Rego et al., "Generation of extreme-ultraviolet beams with time-varying orbital angular momentum," Science 364, eaaw9486 (2019).

10. V. Y. Bazhenov et al., "Laser beams with screw dislocations in their wavefronts," JETP Lett. 52, 429 (1990). 